\title{
Cellular Physiology

\section{Hot Spot Formation in the Nuclear Envelope of Oocytes in Response to Steroids}

\author{
Yvonne Ludwig ${ }^{1}$, Claudia Schafer ${ }^{1}$, Armin Kramer $^{1}$, Lars Albermann², \\ Hans Oberleithner ${ }^{1}$ and Victor Shahin ${ }^{1}$
}

${ }^{1}$ Institute of Physiology II, University of Muenster, ${ }^{2}$ Department of Biochemistry, National University of Ireland Galway

\section{Key Words}

Nuclear envelope - Nuclear pore complex • Nucleocytoplasmic transport • Glucocorticoid • Atomic force microscopy $\cdot$ Ribonucleoprotein

\begin{abstract}
A glucocorticoid-sensitive cell rapidly responds to hormone stimulation with bidirectional exchange of specific macromolecules between cytosol and nucleus. Glucocorticoid-initiated macromolecules (GIMs) must overcome the nuclear envelope (NE) to enter or leave the nucleus. GIM translocation occurs through nuclear pore complexes (NPCs) that span the NE. We investigated the question whether transport of GIMs through NPCs occurs random or involves selected groups of NPCs (hot spots). Glucocorticoid receptors were expressed in Xenopus laevis oocytes and GIM transport was activated by triamcinolone acetonide, a potent synthetic glucocorticoid analogon. Glucocorticoid receptors associated with the NE and the chromatin were identified using western blot analysis and, at single molecule level, atomic force microscopy. Fluorescence-labeled dextran was used to describe passive NE permeability. We observed that after hormone injection (i) small GIMs, most likely
\end{abstract}

\section{KARGER}

Fax +4161306 1234

E-Mail karger@karger.ch www.karger.com
(C) 2006 S. Karger AG, Basel

1015-8987/06/0175-0181\$23.50/0

Accessible online at: www.karger.com/journals/net
GRs, localize within seconds on both sides of the NE. (ii) large GIMs, most likely ribonucleoproteins, localize within minutes on NPCs at the nucleoplasmic side (iii) both small and large GIMs accumulate on selected NPC clusters (iv) NE permeability transiently decreases when GIMs attach to NPCs. We conclude that GIM transport across the nuclear barrier does not randomly take place but is carried out by a selected population of NPCs.

Copyright @ 2006 S. Karger AG, Basel

\section{Introduction}

Nuclear pore complexes (NPCs) are the sole transport pathways for macromolecules between the cytosol and the nucleus [1,2]. Generally, a eukaryotic cell has several millions of NPCs [3-5]. These large protein assemblies (125 MDa) [6] are distributed throughout the entire nuclear envelope (NE) [7, 8] joining its inner (facing the nucleus) and outer (facing the cytosol) nuclear membranes at regular distances. An individual NPC is 
believed to bidirectionally exchange cargo between the cytosol and the nucleus as shown by electron microscopy when RNA-coated gold particles [9] or nuclear localization signals [10] are transported into the nucleus or the cytoplasm of oocytes, respectively. It was also observed that up $97 \%$ of the imaged NPCs are involved in macromolecule transport processes [2,9]. However, it is still unknown whether all NPCs have similar transport capabilities or whether there are distinct functional classes, each involved in transport of specific macromolecules or a group of molecules [11].

In the present study several approaches including molecular biology methods, fluorescence microscopy and atomic force microscopy (AFM) were applied to provide an answer to the above raised question. Cells expressing the glucocorticoid receptor (GR) were exposed to a potent glucocorticoid. This physiological approach triggers an intracellular cascade involving targeting of glucocorticoid initiated macromolecules (GIMs), including proteins like transcription factors and ribonucleoproteins (RNPs), to the cytoplasmic and nucleoplasmic faces of the NE, respectively. As a function of time after glucocorticoid exposure cell nuclei and NE were functionally and structurally analyzed. By a combination of these techniques, the present study describes the pathways that endogenous GIMs take through the NE. Western blotting identified the time point at which GR import into the nucleus, upon glucocorticoid exposure, is initiated. Fluorescence labeled dextrans were applied to measure any changes in NE permeability in response to hormone treatment and to identify the functional opening state of the NPCs. Finally, AFM, a nanotechnique that can resolve individual proteins without complex fixation procedures [12], was used to search for GIMs on either side of the NE spread on a flat substrate. The present study shows that GIM transport is not randomly distributed across NE but occurs at distinct locations.

\section{Materials and Methods}

\section{Preparation of Xenopus laevis oocytes}

Female Xenopus laevis were anaesthetized with $0.1 \%$ ethyl m-aminobenzoate methanesulfonate (Serva, Heidelberg, Germany) and a portion of their ovaries was removed. Stage VI oocytes were dissected from ovary clusters and stored in modified Ringer solution $(87 \mathrm{mmol} /$ $1 \mathrm{NaCl}, 3 \mathrm{mmol} / 1 \mathrm{KCl}, 1.5 \mathrm{mmol} / 1 \mathrm{CaCl}_{2}, 1 \mathrm{mmol} / 1 \mathrm{MgCl}_{2}$, $10 \mathrm{mmol} / 1 \mathrm{HEPES}, 100 \mathrm{U} / 100 \mathrm{mg}$ penicillin/streptomycin, $\mathrm{pH}$ 7.4) and stored at $4{ }^{\circ} \mathrm{C}$ before use.
Expression of rat glucocorticoid receptor (rGR) in Xenopus laevis oocytes

rGR-cRNA was transcribed in vitro from $\mathrm{p} \beta \mathrm{GR} /$ RN3P [13] (kind gift of Ö. Wrange, Karolinska Institute, Stockholm, Sweden) using T3 RNA polymerase (T3 capscribe, Roche, Mannheim, Germany) after linearization with Acc65I (Fermentas, St. Leon-Rot, Germany).10 ng of rGR-cRNA were injected [14] into the cytoplasm and subsequently oocytes were kept in modified Ringer solution [15] at $18^{\circ} \mathrm{C}$ for $72 \mathrm{~h}$.

Oocytes injection, isolation of the nuclei and preparation of nuclear envelopes

$72 \mathrm{~h}$ after rGR-cRNA injection, oocytes were injected with $50 \mathrm{nl}$ solution containing the potent GR agonist triamcinolone acetonide (TA; Sigma, USA). TA concentration in the injection solution ranged $2 \times 10^{-5} \mathrm{~mol} /$ 1 in water, diluted from TA stock solution dissolved: $10^{-2}$ $\mathrm{mol} / \mathrm{l}$ in ethanol). Final TA concentration in a single oocyte was $\sim 1 \mu \mathrm{mol} / 1$ (oocyte volume $\sim 1 \mu \mathrm{l}$ ). Nuclei were isolated at different time points ranging from 90 seconds to 24 hours after oocyte injection. TA-injected oocytes, used in the 24 hours series of experiments, were stored for this time period in TA-containing buffered solution (TA concentration: $1 \mu \mathrm{M}$ ) to maintain a constant intracellular TA concentration prior to the experiments. For nuclear isolation, oocytes were transferred into nuclear isolation medium (NIM) [15]. Nuclei were isolated using precision forceps and then cleared from cytoplasmic debris by drawing the nuclei gently into and out of the tip of an Eppendorf pipette. Nuclei were then picked up with a pipette and transferred to a glass coverslip placed under a stereomicroscope. Subsequently, the chromatin was carefully teased out of the nucleus using sharp needles and the nuclear envelope spread onto a Cell-tak (Biosciences, Bedford, MA USA) coated glass coverslip. For corresponding control experiments, each above mentioned step was similarly performed upon injection of the respective solvent (ethanol in water).

\section{Atomic force microscopy}

To image their cytoplasmic side with AFM, nuclear envelopes of either TA or solvent injected oocytes were spread out with the nucleoplasmic side facing downwards. To image their nucleoplasmic side, however, nuclear envelopes were spread out with the cytoplasmic side facing downwards. Subsequently, in both types of preparations the flat nuclear envelopes, spread on glass, were rinsed with deionized water and air-dried. All procedures were performed at room temperature $\left(\sim 23^{\circ} \mathrm{C}\right)$.

Ludwig/Schafer/Kramer/Albermann/Oberleithner/Shahin 
We used a Bioscope AFM (NanoScope IIIa controller, Digital Instruments, Santa Barbara, CA) equipped with an optical microscope. Aluminum-coated, V-shaped 290 $\mu \mathrm{m}$ long silicon cantilevers with pyramidal tips (tip radius about $10 \mathrm{~nm}$ ) were used. The images were recorded with 512 scan lines per area, at constant force (height mode) in contact mode in air with a scan rate of 3 to $5 \mathrm{~Hz}$. Images were processed using the Nanoscope III software (Digital Instruments). High-resolution imaging of individual NPCs and associated proteins demanded fine-tuning of the scanning process. This comprised mainly the minimizing of the scanning force between tip and sample to values below $3 \mathrm{nN}$. At given times after injection of either TA or solvent into oocytes, about $800 \mu \mathrm{m}^{2}$ (eight areas, each $10 \times 10 \mu \mathrm{m}^{2}$ ) of either the cytoplasmic or the nucleoplasmic sides of the nuclear envelope were visualized with AFM. In each experimental condition 5 nuclear envelopes were analyzed. Data analysis was performed with appropriate software (Nanoscope III software, Digital Instruments, Santa Barbara, USA; SPIP (Scanning probe image processor, Image Metrology, Lyngby, Denmark)).

\section{Analysis of GIMs by AFM}

In order to quantify the size of GIMs, profile analysis was performed in selected areas of the NE. We measured vertical heights of individual GIMs in order to calculate volumes and corresponding molecular masses $[16,17]$.

\section{Western blot analysis}

Nuclear envelopes and chromatins were separated from isolated cell nuclei 40 and 90 seconds after injection of either TA or solvent into GR-expressing oocytes. In each experimental condition, 10 separated nuclear envelopes or chromatins were collected in $200 \mu \mathrm{l}$ reaction tubes. After sedimentation by a short spinning pulse most of the supernatant was removed and $4 \mu 1$ of a $5 \times$ SDSDTT sample loading buffer (0.5 M Tris, 8.5\% SDS, 27.5 $\%$ sucrose, $100 \mathrm{mM}$ DTT, $0.03 \%$ bromphenol blue) was added to the remaining suspension. Proteins were solubilized and denaturated by heating the mixture to $95^{\circ} \mathrm{C}$ for 5 minutes. Then the solution was put on ice for 1 minute and resolved on $7.5 \%$ discontinuous SDSpolyacrylamid gels. Subsequently, proteins were transferred onto nitrocellulose membranes (Schleicher \& Schüll, Dassel, Germany) by electroblotting. Membranes were then incubated in blocking solution (5\% skim milk powder in PBS-T $(1 \mathrm{x}$ PBS, pH. 7.4 containing $0.05 \%$ Tween-20). Subsequently, blots were incubated with a primary antibody directed against GR (SCBT H-300,

Hot Spots in the Nuclear Envelope
Santa Cruz Biotechnology, Heidelberg, Germany) for 1 hour at room temperature or over night at $4^{\circ} \mathrm{C}$. After washing ( $6 \times 5$ minutes with PBS-T) and following incubation with appropriate peroxidase-labeled secondary antibody for 1 hour at room temperature, membranes were washed again. Finally, proteins were detected using a highly sensitive chemiluminescence substrate (Pierce SuperSignal West Femto, Perbio Science, Bonn, Germany). All blots were repeated at least twice yielding similar results.

\section{Confocal fluorescence microscopy}

In a first step, oocytes were microinjected with solvent or TA. Then, after different time periods, nuclei were isolated, mounted to a chamber on the stage of a confocal laser-scanning microscope (CLSM Fluoview, Olympus) and superfused with HEPES buffered solution containing FITC-dextran (fluorescein isothiocyanate; Sigma, St. Louis, MO, USA). The light source of the confocal microscope was an argon/krypton ion laser $\left(\right.$ Omnichrome ${ }^{\circledR}$ ) that generated an excitation wavelength of $488 \mathrm{~nm}$. The underlying principle of exploring passive transport of macromolecules is that a change in NPC conformation is followed by a change in the passive diffusion rate of fluorescence-labeled $20 \mathrm{kDa}$ dextran [18]. Comparison of diffusion rates under different experimental conditions allows conclusions about passive NPC permeability. During the measurement the nucleus was placed in a superfusion chamber (volume $1 \mu 1$ ), which, in combination with a perfusor, allows permanent superfusion and fast changes in perfusion solutions. To prevent the cell nucleus from moving, it was kept in place by using Cell-tak (Biosciences, Bedford, USA). Diffusion of 20 kDa FITC- dextran across the nuclear envelope was measured and analyzed with confocal fluorescence microscopy as previously described [18]. Ninety seconds, $3,5,10,20$ minutes and 24 hours after injection of $50 \mathrm{nl}$ of either TA or solvent into GR expressing oocytes, cell nuclei were isolated and collected in NIM. Subsequently, nuclei were incubated for 2 minutes in NIM containing 5 $\mu \mathrm{mol} / 120 \mathrm{kDa}$ FITC-dextran and then placed on Cell-tak in a perfusion chamber to detect their fluorescence. To test for TA-induced transcription 20 minutes after injection, $5 \times 10^{-7} \mathrm{M}$ of the transcription inhibitor actinomycin D (Sigma, USA) was co-injected with $2 \times 10^{-5} \mathrm{M}$ TA (injection volume $=50 \mathrm{nl}$ ).

\section{Statistics}

Data are presented as mean values \pm standard error of the mean (SEM). Each triamcinolone acetonide 


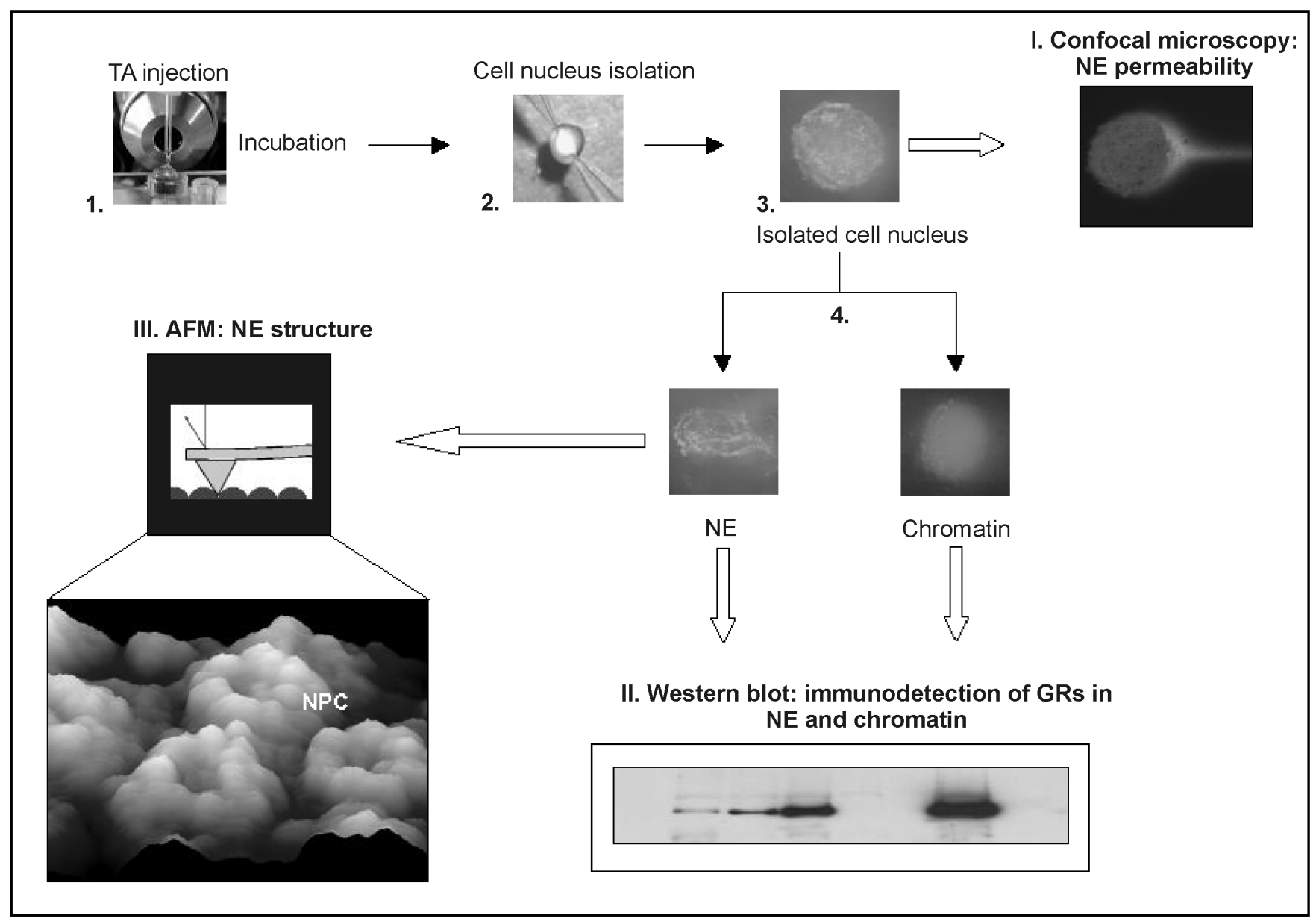

Fig. 1. Experimental approach, in which cell nuclei and nuclear envelopes (NEs) can be structurally and functionally investigated as a function of time after injection of the glucocorticoid receptor (GR) agonist, triamcinolone acetonide (TA), into GRexpressing Xenopus laevis oocytes. AFM = Atomic force microscopy; NPC = nuclear pore complex.

experimental condition was statistically compared with its respective control. Statistical analysis was performed by unpaired Student's t-test. An asterisk $(*)$ indicates a significant difference of $P<0.01$ or less. The calculated $P$ values are mentioned in the respective figure legends.

\section{Results}

A scheme of the experimental approach of the present study is displayed in figure 1. A highly potent GR agonist, triamcinolone acetonide (TA), was injected into GR-expressing Xenopus laevis oocytes. AFM was applied to structurally investigate the transport pathways of glucocorticoid initiated macromolecules (GIMs) as a function of time after TA injection. Measurements of passive nuclear envelope permeability using fluorescence- labeled $20 \mathrm{kDa}$ dextran paralleled AFM investigations. According to a previous study [19] the permeability measurements served as a functional indicator for the existence of GIMs at the nuclear envelope. GIMs were divided (based on shape and size) into small GIMs which were visualized on either side of the NE within seconds and large GIMs visualized only on the nucleoplasmic side of the NE, as early as 20 minutes after injection.

\section{Passive NE permeability}

We investigated the effect of injecting either solvent or TA into the cytosol of GR-expressing oocytes on passive NE permeability using $20 \mathrm{kDa}$ FITC-dextran. Measurements were performed between 90 seconds and 20 minutes after injection. First, these measurements were compared with controls (non-injected, non-GR-expressing oocytes). The nuclei of these control oocytes $(n=56)$

Ludwig/Schafer/Kramer/Albermann/Oberleithner/Shahin 


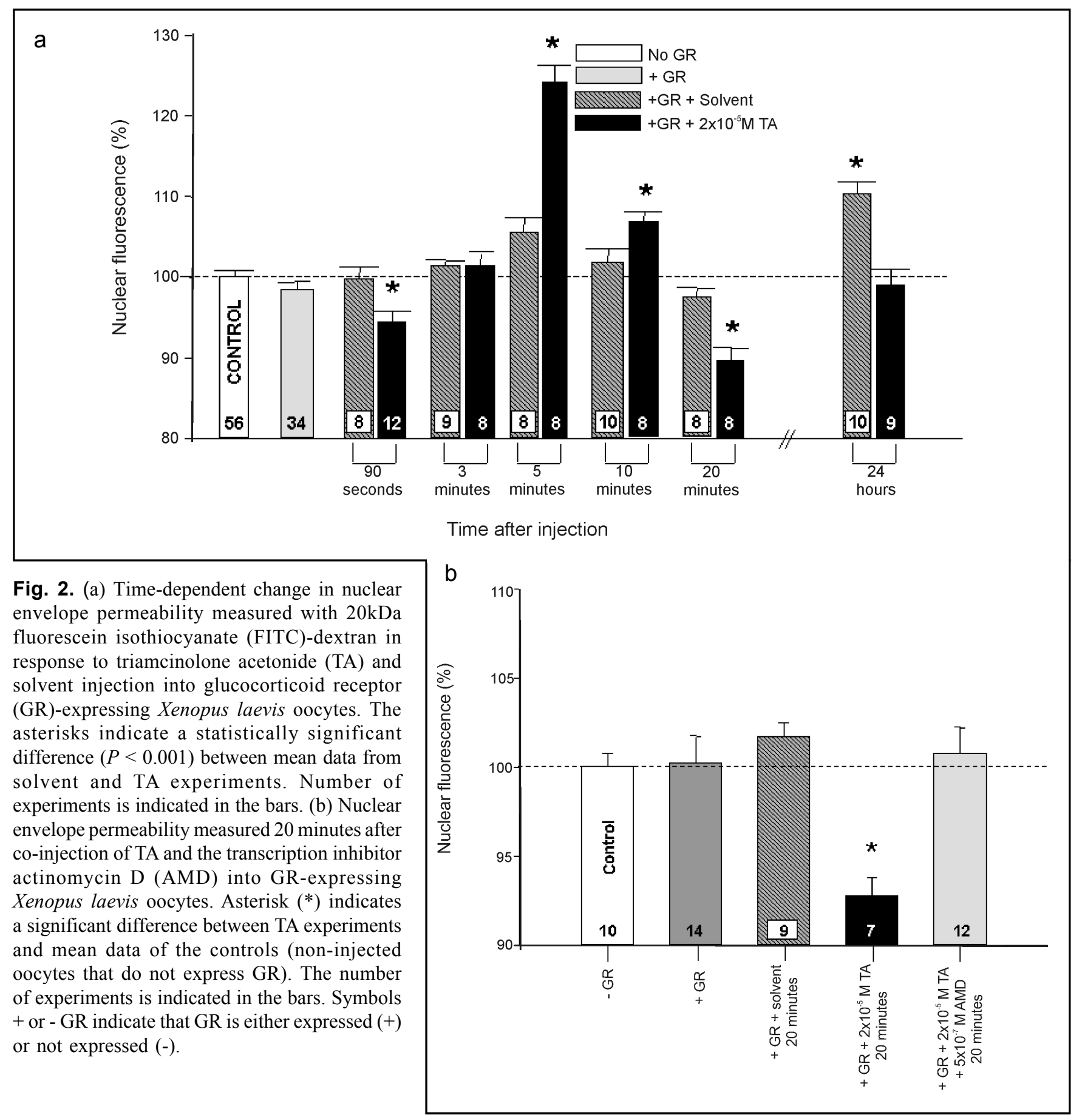

exhibited a 'standard' level of nuclear fluorescence (100 $\%=$ control nuclear fluorescence). Nuclear fluorescence of solvent- or TA-injected GR-expressing oocytes was related to this control fluorescence. As seen in figure 2a, following solvent injection, no remarkable change of nuclear fluorescence was detectable over the entire time scale. In contrast, nuclear fluorescence significantly

Hot Spots in the Nuclear Envelope decreased $(94.5 \pm 1.40 \%, n=12$ nuclei) within 90 seconds after TA injection. This initial decrease was followed by a remarkable increase $(124.2 \pm 2.07 \%, n=$ 8 nuclei) of nuclear fluorescence observed 5 minutes upon TA-injection. Subsequently, nuclear fluorescence gradually decreased reaching a minimum value 20 minutes after injection $(89.7 \pm 1.63 \%, n=8$ nuclei). To test for a long- 


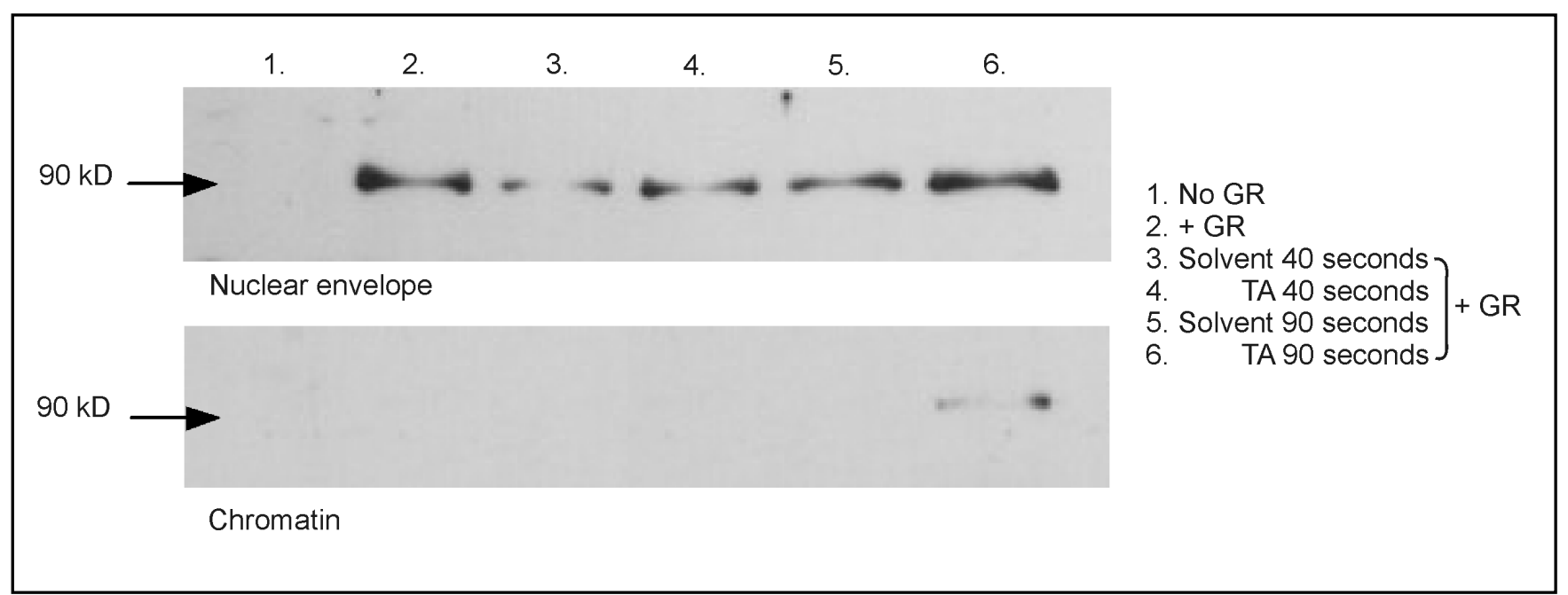

Fig. 3. Western blot analysis of rat glucocorticoid receptor (rGR) in nuclear envelopes (upper panel) and chromatin (lower panel) of rGR-expressing Xenopus laevis oocytes 40 and 90 seconds after triamcinolone acetonide (TA) or solvent injection into oocytes. Non TA-injected oocytes that either did not express rGR (lane 1) or did express rGR (lane 2) served as controls.

term effect of TA exposure we incubated oocytes after initial TA injection in $1 \mu \mathrm{M}$ TA and performed nuclear permeability measurements 24 hours later. In parallel, we did similar experiments using solvent instead of TA. As indicated in figure 2a long-term incubation in solvent led to an increase in permeability above the initial values (110.74 $\pm 1.59 \%, n=10$ nuclei). Obviously, the NE became leaky over this long ( 24 hours) incubation period. In contrast, long-term TA exposure prevented this permeability increase $(99.1 \pm 1.95 \%, n=9$ nuclei).

Finally we tested whether the TA-induced decrease in passive macromolecule permeability was mediated by transcriptional processes in the cell nucleus. We coinjected TA with actinomycin $\mathrm{D}$, a potent transcription inhibitor and then measured NE permeability 20 minutes later. Indeed, application of actinomycin D prevented the decrease in nuclear fluorescence (figure $2 b$ ).

\section{Western blot analysis}

The decrease in nuclear permeability 90 seconds after TA injection was likely to be caused by GIMs attached to NPCs of the NE. To identify GRs among GIMs we performed western blot analysis experiments. NEs and chromatins of GR-expressing oocytes were isolated and analyzed 40 and 90 seconds after injection of either solvent or TA. Upon treatment with a primary antibody directed against GR, we tested for the presence of a $\sim 90 \mathrm{kDa}$ band on western blots [14]. As shown in figure 3, freshly isolated NEs and chromatins of notinjected, not-GR-expressing oocytes did not exhibit a visible band (lane 1). NEs of GR-expressing oocytes, but not injected with TA, contained a small amount of GR indicated by a band at $\sim 90 \mathrm{kDa}$ (lane 2). 40 seconds after solvent injection (lanes 3 and 5) a GR-related band was found only in isolated NEs but not in the chromatin fractions. Identical findings were observed also 90 seconds after solvent injection. Similar to solvent, 40 seconds after TA injection, a small amount of GR was detected in the NE but not in the chromatin fraction (lane 4). Furthermore, and opposite to the 40 seconds experiment, 90 seconds after TA injection also chromatin revealed a clear GR band at $90 \mathrm{kDa}$ (lane 6).

\section{Atomic force microscopy}

As seen in figures $4 \mathrm{a}$ ( 90 seconds) and $4 \mathrm{~b}$ ( 24 hours), after solvent injection NPCs appeared as smooth rings at the cytoplasmic side of the NE. NPCs were predominantly free of transport cargo. Similarly, NPCs of the nucleoplasmic side of the NE were found almost free of cargo at the same conditions ( 90 seconds, 20 minutes, 24 hours ; fig. $4 \mathrm{c}, \mathrm{d}$ and e). In contrast to solvent treatment, remarkable changes took place on both sides of the NE 90 seconds, 20 minutes and 24 hours after TA injection (fig. 5). Figures 5a and 5b display representative AFM images of the cytoplasmic side of the NE 90 seconds and 24 hours after TA injection into GR-expressing oocytes. Obviously, NPCs clustering occurs rather than equal distribution of NPCs. In agreement with western blot analysis that showed GR import 90 seconds after TA injection, the observed macromolecules on the NPCs most

Ludwig/Schafer/Kramer/Albermann/Oberleithner/Shahin 
Fig. 4. Atomic force microscopy images of the cytoand nucleoplasmic faces of glucocorticoid receptor (GR)expressing Xenopus laevis oocytes after solvent injection. Cytoplasmic face of the nuclear envelope 90 seconds (a) and 24 hours (b) after solvent injection. Nucleoplasmic face of the nuclear envelope 90 seconds (c), 20 minutes (d) and 24 hours (e) after solvent injection. Lower images correspond to the nuclear pores marked in the upper images.
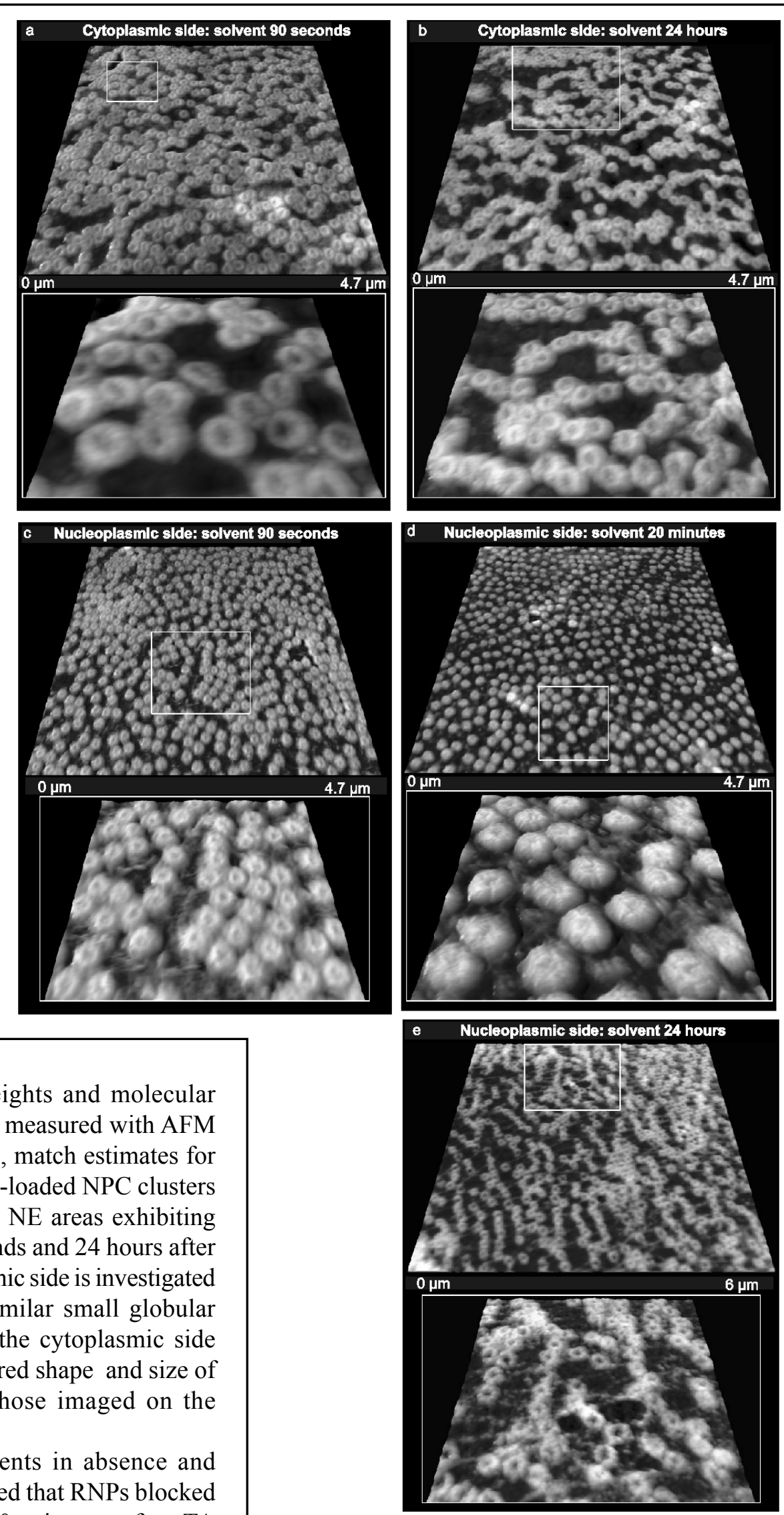

likely were GIMs. Molecular heights and molecular masses of the detected molecules, measured with AFM according to previous studies [17], match estimates for GRs (data not shown). Small GIM-loaded NPC clusters were usually surrounded by large NE areas exhibiting cargo-free, resting NPCs, 90 seconds and 24 hours after TA injection. When the nucleoplasmic side is investigated 90 seconds after TA injection, similar small globular macromolecules as observed on the cytoplasmic side could be detected (fig. 5c). Measured shape and size of the latter cargo clearly match those imaged on the cytoplasmic side of the NE.

NE permeability measurements in absence and presence of actinomycin D indicated that RNPs blocked the NPC mediated pathways 20 minutes after TA 
Fig. 5. Atomic force microscopy images of the cyto- and nucleoplasmic faces of glucocorticoid receptor (GR)expressing Xenopus laevis oocytes after triamcinolone acetonide (TA) injection. Cytoplasmic face of the nuclear envelope 90 seconds (a) and 24 hours (b) after injection exhibits NPC clusters ('hot-spots' covered with small GIMs) and areas with resting nuclear pores (free of cargo). Nucleoplasmic face of the nuclear envelope 90 seconds (pores covered with small GIMs) (c), 20 minutes (pores covered with large GIMs) (d) and 24 hours (pores covered with large GIMs) (e) after injection exhibit either resting pores or transporting clusters. Lower images correspond to the nuclear pores marked in the upper images.
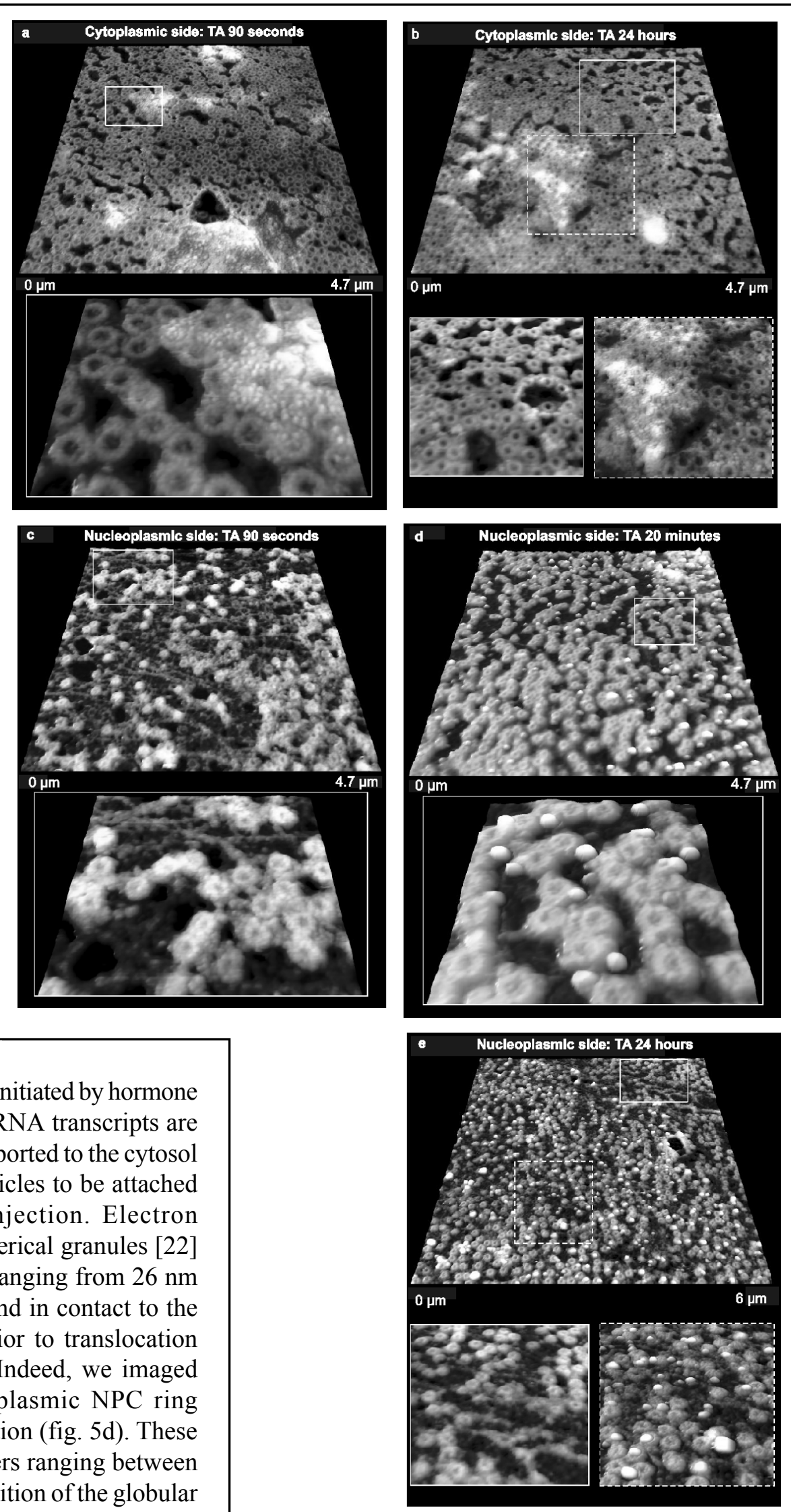

injection. When gene transcription is initiated by hormone injection, newly-synthesized pre-mRNA transcripts are being packed as RNPs [20] and transported to the cytosol [21]. Hence, we expected RNP particles to be attached to NPCs 20 minutes after TA injection. Electron microscopy visualized RNPs as spherical granules [22] or rings [23] of variable diameters ranging from $26 \mathrm{~nm}$ [21] to $50 \mathrm{~nm}$ [22]. RNPs were found in contact to the nucleoplasmic NPC basket [22] prior to translocation through the NPC central channel. Indeed, we imaged globular structures at the nucleoplasmic NPC ring periphery 20 minutes after TA injection (fig. 5d). These globular structures revealed diameters ranging between 20 to $60 \mathrm{~nm}$. Shape, diameter and position of the globular structures strongly argue for RNP particles. Also after

$$
\text { Ludwig/Schafer/Kramer/Albermann/Oberleithner/Shahin }
$$




\begin{tabular}{|c|c|c|c|c|c|}
\hline \multirow{2}{*}{$\begin{array}{l}\text { Steroid } \\
\text { exposure }\end{array}$} & \multicolumn{5}{|c|}{ Methods } \\
\hline & $\begin{array}{c}\text { Electrical } \\
\text { conductivity }\end{array}$ & Permeability & AFM & Western blot & Interpretation \\
\hline $90 \mathrm{~s}$ & n.m. & Decrease for $20 \mathrm{kDa}$ Dextran ${ }^{[*]}$ & $\begin{array}{l}\text { Pore dilation } \\
\text { Single molecules cover NE }{ }^{[17]} \\
\text { Different patterns of NE: } \\
\text { Transporting NPC clusters (hot spots) } \\
\text { Resting pores } \\
\text { Small molecules attach to hot spots }{ }^{\left[{ }^{*}\right]}\end{array}$ & $\begin{array}{l}\text { rGRs accumulate } \\
\text { in nuclei }{ }^{[17]} \\
\text { rGRs accumulate } \\
\text { in chromatin }{ }^{[*]}\end{array}$ & $\begin{array}{l}\text { Cargo attach to NPC filaments } \\
\text { Pores dilate and form clusters } \\
\text { Earliest time point of cargo } \\
\text { import at hot spots }\end{array}$ \\
\hline 2 to $3 \mathrm{~min}$ & Decrease $^{[19]}$ & Basal level ${ }^{[*]}$ & $\begin{array}{l}\text { NPCs decorated with } \\
\text { macromolecules } \\
\text { Further pore dilation } \\
\text { Single molecules at NPC rim } \\
\text { and in central channel }{ }^{[17]}\end{array}$ & n.m. & $\begin{array}{l}\text { Cargo occludes peripheral } \\
\text { hannels } \\
\text { Cargo import }\end{array}$ \\
\hline $5 \mathrm{~min}$ & Increase $^{[19,24]}$ & Increase for $20 \mathrm{kDa}$ Dextran ${ }^{[24, *]}$ & $\begin{array}{l}\text { Pore dilation } \\
\text { Different patterns of NE: } \\
\text { Pattern I: Low NPC density } \\
\text { Pattern II: High NPC density } \\
\text { No molecules detecable } \\
\text { Hydrophobicity of NE increases }{ }^{[24]} \\
\text { Pore dilation } \\
\text { Detection of giant pores }^{[30]}\end{array}$ & n.m. & $\begin{array}{l}\text { Peak of cargo import } \\
\text { Cargo interacts with FG-repeats } \\
\text { leading to pore dilation } \\
\text { Pore dilation is a conformational } \\
\text { pre-state for transient giant pores }\end{array}$ \\
\hline $\begin{array}{l}10 \text { and } 11 \\
\min \end{array}$ & Increase $^{[19,30]}$ & $\begin{array}{l}\text { Import and export increase } \\
\text { for } 10 \mathrm{kDa} \text { Dextran } \\
\text { Increase of nuclear volume }{ }^{[29]} \\
\text { Increase for } 77 \mathrm{kDa} \text { Dextran } \\
\text { Increase for } 20 \mathrm{kDa} \text { Dextran } \\
{ }^{[*]}\end{array}$ & $\begin{array}{l}\text { Pore dilation } \\
\text { Detection of giant pores }\end{array}$ & n.m. & $\begin{array}{l}\text { Cargo import } \\
\text { Giant pore formation }\end{array}$ \\
\hline $\begin{array}{l}18 \text { to } 20 \\
\min \end{array}$ & Decrease $^{[19]}$ & $\begin{array}{l}\text { Decrease for } 20 \mathrm{kDa} \text { Dextran } \\
\text { AMD prevents decrease }{ }^{[*]}\end{array}$ & $\begin{array}{l}\text { Macromolecules plug NPC } \\
\text { central channels }{ }^{[19]} \\
\text { Different NPC patterns of NE: } \\
\text { Transporting NPC clusters (hot spots) } \\
\text { Resting pores } \\
\text { Large molecules attach to hot spots }{ }^{\left[{ }^{*}\right]}\end{array}$ & n.m. & $\begin{array}{l}\text { Earliest time point of RNP } \\
\text { export at hot spots }\end{array}$ \\
\hline $24 \mathrm{~h}$ & $\mathrm{n} . \mathrm{m}$. & Decrease for $20 \mathrm{kDa}$ Dextran ${ }^{\left[{ }^{*}\right]}$ & $\begin{array}{l}\text { Different NPC patterns of NE: } \\
\text { NPC clusters (hot spots) } \\
\text { Resting pores } \\
\text { Small molecules attach to } \\
\text { hot spots on the cytoplasmic NE } \\
\text { Large molecules attach to } \\
\text { hot spots on the nucleoplasmic NE }{ }^{[*]}\end{array}$ & n.m. & $\begin{array}{l}\text { Import and export at hot spots } \\
\text { If the stimulus is strong } \\
\text { resting NPCs } \\
\text { will be recruited from stores }\end{array}$ \\
\hline
\end{tabular}

Table 1. Time course of steroid-induced changes of the nuclear envelope. Abbreviations:AMD $=$ actinomycin $\mathrm{D}$; NE $=$ nuclear envelope; $\mathrm{n} . \mathrm{m} .=$ not measured; $\mathrm{NPC}=$ nuclear pore complex; $\mathrm{rGR}=$ rat glucocorticoid receptor; $\mathrm{RNP}=$ ribonucleoprotein $[*]=$ this paper; steroid exposure $=$ time after steroid injection into Xenopus leavis oocytes

long-term stimulation ( 24 hours) the nucleoplasmic side of the NE exhibited two different patterns, areas of transporting NPC clusters (hot spots) and resting pores. In addition, large GIMs gathered in hot spots on the nucleoplasmic side of the NE (fig. 5e).

\section{Discussion}

The present study aims to elucidate the pathways that steroid-triggered macromolecules take through the

Hot Spots in the Nuclear Envelope nuclear envelope. Passive macromolecule NE permeability characterizes the functional opening state of NPCs $[19,24]$ that represent the major transport routes between cytosol and nucleus. Translocation of cargo through NPCs results in a transient decrease in permeability [19]. This permeability change was used as a valuable information on the functional state of NPCs at a given time after TA injection. Actually, this permeability change most likely indicated the interaction of GIMs with NPCs as demonstrated previously [19]. The present study shows that the decreases of NE permeability coincide 
with the accumulation of GIMs on both sides of the NE. We assume that the early decrease of passive NE permeability is due to GIM import, in particular GRs. GIMs detected at this early stage match heights $(\sim 7 \mathrm{~nm})$ and molecular masses $(\sim 90 \mathrm{kDa})$ of activated GRs. In addition, western blot analysis detected GRs in the chromatin 90 seconds after TA stimulation, i.e. at the same time when transported GIMs were detected by AFM. Unfortunately, our current technical approach does not allow to identify GRs among other proteins similar in size at the single molecule level. Most likely, GIMs are composed of various molecule species and only one of them is the GR. Single GR detection would need the application of a more sophisticated molecule recognition technique as developed by Hinterdorfer and coworkers [25]. With this elegant AFM-based technique GRs could not only be identified at the NE surface but also quantified at single molecule level.

Within 90 seconds after TA injection, GIMs arrive at the NE (fig. 5a and 5c). They attach to the NPCs and block passive macromolecule permeability. Rapid changes in NE permeability accompanying hormone mediated macromolecule transport across NPCs is a well-known phenomenon [26]. Another transient decrease in permeability is found 20 minutes after hormone activation. This 'late response' coincides with the appearance of large GIMs attached to the nucleoplasmic side of individual NPCs. From the time lag after hormone injection and from the size of the particles we assumed that they represent newly synthesized mRNA packed as RNPs, on their way out of the nucleus. This assumption is strongly supported by the observation that actinomycin $\mathrm{D}$, a transcription inhibitor, eliminates this late response. Dimensions of these large GIMs match with those reported for RNPs [21, 22, 27] and also with previous AFM measurements of plugs that were found inside NPC central channels at similar time after aldosterone stimulation [19]. The long-term experiments (24 hours) of the present study support the view that the large GIMs, found on the nucleoplasmic side of the NE, were responsible for the decrease in NE permeability. Whenever large GIMs could be detected by AFM, either 20 minutes or 24 hours after beginning of TA stimulation, we found a decreased permeability, when compared with the corresponding controls (solvent) (fig. 2a). Taken together, the data indicate that GIM export and GIM import are always associated with a permeability decrease of the nuclear barrier.

Nuclei of mature Xenopus laevis oocytes are equipped with about 40 millions of NPCs [28]. So far, it has been unclear whether all NPCs participate in transport and whether NPCs are 'chosen at random' to transfer macromolecules in and out of the nucleus in response to a hormone stimulus. Obviously, this is not the case. The present study indicates that there are hot spots in the NE where GIM transport occurs. Hot spots represent clusters of possibly several hundreds of transporting NPCs. Since both small and large GIMs are organized in such clusters it is tempting to assume that import of GRs and export of RNPs occur at the same NE sites. Most likely, a major portion of the NE remains in a resting state while a 'predetermined', small portion of the NE becomes active upon hormone stimulation. Unfortunately, we can only give a qualitative description of these phenomena and cannot quantify e.g. the number of hot spots or the number of NPCs organized within a cluster. We also do not know much about the kinetics of cluster formation. Such questions, though interesting to ask, are hardly to answer since most likely number and dimensions of hot spots will strongly depend upon the stimulus. We assume that resting NPCs in the neighborhood of hots spots will be recruited if the stimulus is strong and persisting.

Table 1 summarizes the results obtained in previous experiments $[17,19,24,29,30]$ and in the present study. It documents the chronological sequence of cargo import into the nucleus upon steroid stimulation. Interestingly, NPCs change conformation prior to physical interaction with the cargo itself. After cargo binding to NPC filaments or to specific binding sites, nuclear pores dilate [17]. 90 seconds after steroid injection NPCs form clusters and cargo import starts at 'hot spots' of the NE. Strong signs of import are observed 5 minutes after stimulation. The hydrophobic cargo interacts with FG-repeats inside the central channel of NPCs and initiates an expansion of the NPC opening [24]. This dilation may be a conformational pre-state for transient giant pore formation (nuclear pores with a diameter of about $300 \mathrm{~nm}$ ) which appears 5 to 11 minutes after steroid injection [30]. The earliest time point of RNP export can be observed 18 to 20 minutes after stimulation [19]. In this study, we could show that this occurs at hot spots on the NE.

GIM transport organized in clusters within the NE is possibly useful to specifically target gene loci in the nucleus. It is well known that gene order in the nucleus is not random. Genes that are expressed in a similar and/or coordinated way, are often closely located to each other [31]. Lee and Sonnhammer [32] found out that functionally related genes are clustered. It is tempting to speculate that selected NPC clusters drive translocated GRs directly to adjacent specific chromatin sites where transcriptional

Ludwig/Schafer/Kramer/Albermann/Oberleithner/Shahin 
processes are initiated. Ris showed [33] that the nucleoplasmic faces of NPCs are interconnected by 50 $\mathrm{nm}$ channels formed from eight $6 \mathrm{~nm}$ filaments. Channels from different NPCs merge to form common channels that then run deeper into the nucleus. It is also known that in the interphase nucleus, individual chromosomes occupy distinct, non-overlapping regions [34]. One consequence is that the chromosomes segregate the nuclear interior into inter-chromosomal channels. Such channels provide a continuous pathway for molecule trafficking from the chromosomes to the nuclear periphery [35]. In conclusion, hormone stimulated macromolecule transport most likely occurs at distinct spots in the nuclear envelope, possibly close to the chromatin sites of specific gene expression.
Abbreviations

AFM (atomic force microscopy); AMD (actinomycin D); FITC (fluoresceine isothiocyanate); GIM (glucocorticoid initiated macromolecules); rGR (rat glucocorticoid receptor); NE (nuclear envelope); NIM (nuclear isolation medium); NPC (nuclear pore complex); RNP (ribonucleoproteins); SEM (standard error of the mean); TA (triamcinolone acetonide).

\section{Acknowledgements}

The authors gratefully acknowledge the excellent technical assistance of Barbara Windoffer. This study was supported by the Deutsche Forschungsgemeinschaft (SFB 629-A6) and by the EU grant "Tips4cells".

\section{References}

Allen TD, Cronshaw JM, Bagley S, $>8$ Kiseleva E ,Goldberg MW: The nuclear pore complex: mediator of translocation between nucleus and cytoplasm. J Cell Sci 2000;113:1651-1659.

$\checkmark 2$ Gorlich D, Mattaj IW: Nucleoplasmic transport. Science 1996;271:1513-1518.

3 Akey CW, Radermacher M. Architecture $>9$ of the Xenopus nuclear pore complex revealed by three-dimensional cryoelectron microscopy: J Cell Biol 1993;122:1-19.

4 Forbes DJ: Structure and function of the nuclear pore complex. Annu Rev Cell Biol 1992;8:495-527.

5 Pante N, Aebi U: The nuclear pore $>11$ complex. J Cell Biol 1993;122:977-984. Reichelt R, Holzenbur A, Buhle EL, Jarnik M, Engel A, Aebi U: Correlation between structure and mass distribution of the nuclear pore complex and if distinct pore complex components. J Cell Biol 1990;110:883-894

7 Gerace L: Functional organization of the nuclear envelope. Annu Rev Cell Biol 1988;4:335-374.
Goldberg MW, Allen TD: High resolution scanning electron microscopy of the nuclear envelope: demonstration of a new, regular, fibrous lattice attached to the baskets of nucleoplasmic face of the nuclear pores. J Cell Biol 1992;119:1429-1440.

Dworetzky SI, Feldherr CM: Translocation of RNA-coated gold particles through the nuclear pores of oocytes. J Cell Biol 1988;106:575-584. Newmeyer DD, Forbes DJ: Nuclear $>16$ import can be separated into distinct steps in vitro: nuclear pore binding and translocation. Cell 1988;52:641-653.

Feldherr CM, Kallenbach E, Schultz N: Movement of a karyophilic protein through the nuclear pores of oocytes. $J$ Cell Biol 1984;99:2216-2222.

12 Barrera NP, Herbert P, Henderson RM, Martin IL, Edwardson JM: Atomic force microscopy reveals the stoichiometry and subunit arrangement of 5-HT3 receptors. Proc Natl Acad Sci U S A 2005; 102:12595-12600

13 Belikov S, Gelius B, Almouzni, Wrange $\mathrm{O}$ : Hormone activation induces nucleosome positioning in vivo. Embo $\mathrm{J}$ 2000;19:1023-1033.
Albermann L, Shahin V, Ludwig Y, Schafer C, Schillers H, Oberleithner H: Evidence for Importin alpha Independent Nuclear Translocation of Glucocorticoid Receptors in Xenopus laevis Oocytes. Cell Physiol Biochem 2004;14:343-350.

-15 Shahin V, Danker T, Enss K, Ossig R, Oberleithner $\mathrm{H}$ : Evidence for $\mathrm{Ca} 2+-$ and ATP-sensitive peripheral channels in nuclear pore complexes. FASEB J 2001;15:1895-1901.

Schneider SW, Larmer J, Henderson RM, Oberleithner $\mathrm{H}$ : Molecular weights of individual proteins correlate with molecular volumes measured by atomic force microscopy. Pflugers Arch 1998;435:362-367.

Shahin V, Albermann L, Schillers H, Kastrup L, Schafer C, Ludwig Y, Stock C, Oberleithner H: Steroids dilate nuclear pores imaged with atomic force microscopy. J Cell Physiol. 2004;202:591-601.

Enss K, Danker T, Schlune A, Buchholz I, Oberleithner H: Passive transport of macromolecules through Xenopus laevis nuclear envelope. J Membr Biol 2003;196:147-155. 
19 Schafer C, Shahin V, Albermann L, Hug MJ, Reinhardt J, Schillers H, Schneider SW, Oberleithner $\mathrm{H}$ : Aldosterone signaling pathway across the nuclear envelope. Proc Natl Acad Sci U S A 2002;99:7154-7159.

20 Dreyfuss G, Matunis MJ, Pinol-Roma S, and Burd CG: hnRNP proteins and the biogenesis of mRNA. Annu Rev Biochem 1993;62:289-321.

21 Kiseleva E, Goldberg MW, Allen TD, Akey CW: Active nuclear pore complexes in Chironomus: visualization of transporter configurations related to mRNP export. J Cell Sci 1998;111:223236.

22 Stevens BJ, Swift H: RNA transport from nucleus to cytoplasm in Chironomus salivary glands. J Cell Biol 1966;31:5577.

23 Mehlin H, Daneholt B, Skoglund U: Structural interaction between the nuclea pore complex and a specific translocating RNP particle. J Cell Biol 1995;129:12051216.

24 Shahin V, Ludwig Y, Schafer C, Nikova D, Oberleithner H: Glucocorticoids remodel nuclear envelope structure and permeability. J Cell Sci 2005;118:28812889.
25 Hinterdorfer P, Baumgartner W, Gruber HJ, Schilcher K, Schindler H: Detection and localization of individual antibodyantigen recognition events by atomic force microscopy. Proc Natl Acad Sci U S A 1996;93:3477-3481.

Mazzanti M, Bustamante JO, Oberleithner H: Electrical dimension of the nuclear envelope. Physiol Rev 2001;81:1-19.

Sperling R, Koster AJ, Melamed-Bessudo C, Rubinstein A, Angenitzki M, Berkovitch-Yellin Z, Sperling J: Threedimensional image reconstruction of large nuclear RNP (lnRNP) particles by automated electron tomography. J Mol Biol 1997;267:570-583.

Maul GG: The nuclear and the cytoplasmic pore complex: structure, dynamics, distribution, and evolution. Int. Rev. Cytol Suppl 1977;6:75-186. Buchholz I, Enss K, Schafer C, Schlune A, Shahin V, Oberleithner H: Transient permeability leak of nuclear envelope induced by aldosterone. J Membr Biol 2004;199:135-141.
Kastrup L, Oberleithner H, Ludwig Y, Schafer C, Shahin V: Nuclear envelope barrier leak induced by dexamethasone. J Cell Physiol 2006;206:428-434.

Hurst LD, Pal C, Lercher MJ: The evolutionary dynamics of eukaryotic gene order. Nat Rev Genet 2004;5:299310.

Lee JM, Sonnhammer EL: Genomic gene clustering analysis of pathways in eukaryotes. Genome Res 2003;13:87582.

Ris H: High-resolution field-emission scanning electron microscopy of nuclear pore complex. Scanning 1997;19:368375.

34 Kurz A, Lampel S, Nickolenko, JE, Bradl J, Benner A, Zirbel RM, Cremer T, Lichter P: Active and inactive genes localize preferentially in the periphery of chromosome territories. J Cell Biol 1996;135:1195-1205.

Bridger JM, Herrmann H, Munkel C, Lichter P: Identification of an interchromosomal compartment by polymerization of nuclear-targeted vimentin. J Cell Sci 1998;111:1241-53. 